\title{
Spot the difference: Solving the puzzle of hidden pictures in the lizard genome for identification of regeneration factors
}

\author{
Jin Woong Chung ${ }^{*}$ \\ Department of Biological Science, Dong-A University, Busan 49315, Korea
}

\begin{abstract}
All living things share some common life processes, such as growth and reproduction, and have the ability to respond to their environment. However, each type of organism has its own specialized way of managing biological events. Genetic sequences determine phenotypic and physiological traits. Based on genetic information, comparative genomics has been used to delineate the differences and similarities between various genomes, and significant progress has been made in understanding regenerative biology by comparing the genomes of a variety of lower animal models of regeneration, such as planaria, zebra fish, and newts. However, the genome of lizards has been relatively ignored until recently, even though lizards have been studied as an excellent amniote model of tissue regeneration. Very recently, whole genome sequences of lizards have been uncovered, and several attempts have been made to find regeneration factors based on genetic information. In this article, recent advances in comparative analysis of the lizard genome are introduced, and their biological implications and putative applications for regenerative medicine and stem cell biology are discussed. [BMB Reports 2016; 49(5): 249-254]
\end{abstract}

\section{INTRODUCTION}

Regardless of whether they believe in evolutionary theory or creationism, nobody would argue against the idea that humans are the cleverest creatures among all living organisms. However, humans lack certain abilities that lower animals possess; humans cannot fly like eagles, cannot swim like dolphins, and cannot run as fast as cheetahs. Because humans have been longing for such abilities, we have developed various machines that provide us with them, such as airplanes, ships, and cars.

*Corresponding author. Tel: +82-51-200-7270; Fax: +82-51-2007269; E-mail: jwchung@dau.ac.kr

http://dx.doi.org/10.5483/BMBRep.2016.49.5.045

Received 4 March 2016

Keywords: Comparative genomics, Dedifferentiation, Lizard, Regeneration, Stem cells
The desire to mimic animals facilitated the development of biomimetics, the study of designing human-made apparatuses that imitate nature, and humans can now manufacture a variety of useful things, such as solar cells that mimic the leaves of plants, steel fibers inspired by spider webs, infrangible ceramics like clam shells, and computers that send signals like living cells. Although biomimetic approaches led to the invention of various tools that allow humans to imitate the behaviors of animals or plants, there are still many things that humans, by themselves, cannot physiologically do. Of such things, regeneration has been thought of as a thing that humans will never possess.

Many lower animals have the capacity for spontaneous tissue regeneration after injury, a property that is lacking in humans except for limited liver regeneration (1). In general, animals with a lower evolutionary status have a higher ability to regenerate parts of themselves. As an example, many invertebrates such as planaria can regrow their entire bodies from as little as one-tenth of the original parts (2). Lower vertebrates such as zebrafish, newts, and lizards can also regenerate almost every tissue (3), while humans and other mammals cannot, although limited regeneration in mammals has been reported $(1,4)$. As a matter of fact, lizards are the only amniotic vertebrae that possess a self-regenerative capacity. Thus, it seems that such regenerative abilities might have been lost during evolution, probably when birds and mammals evolved from early reptiles, although enough direct lines of evidence do not exist to support that statement. The lack of evidence might, at least partially, be due to insufficient information about the genomic sequences of lizards despite the development of modern genomic technologies. Very recently, whole lizard genomes have been sequenced, and several genomic or proteomic attempts have been made to identify the factors responsible for regeneration. Genetic studies have produced valuable information on topics of interest that can be applied in various fields, including agriculture, industrial biotechnology, and medicine. Thus, in this article, recent advances in lizard genome sequencing and a comparative analysis of the regenerative mechanisms in lizards are introduced, and possible strategies for the development of regenerative medicine in humans are discussed. 


\section{EVOLUTIONARY AND GENOMIC CHARACTERISTICS OF LIZARDS}

Reptiles first evolved from amphibians in the late Carboniferous period about 320 million years ago, when they evolutionarily parted ways with other amoniotes such as birds and mammals (5). Evolution is driven by a change in the gene pool of a population of organisms over time. Ultimately, these genetic changes constitute unique genomes and give organisms specific features, genetically and physiologically. The genomic sequences of a variety of organisms, from simple microorganisms to higher vertebrates, including humans, have been uncovered. However, the genomes of reptiles, especially lizards, seem to have been ignored until recently, even though lizards are regarded as important models for developmental biology, neurobiology, endocrinology, behavioral biology, and cancer biology (6-9).

The lizard genome was first published by Alfoldi et al. in 2011 (10). Those authors sequenced and assembled the genome of the green anole lizard Anolis carolinensis, which has 18 chromosomes, including 6 pairs of macrochromosomes and 12 pairs of microchromosomes with a total size of 1.78 $\mathrm{Gb}$; for comparison, the chicken genome contains 28 pairs of microchromosomes $(11,12)$. Microchromosomes are mainly found in birds, and also in certain reptiles, fishes, and amphibians, whereas they seem to be absent in mammals. The presence of microchromosomes has made genomic study of the lower animals difficult because of their small size and poor condensation (13). Despite the small size of microchromosomes, however, they have been estimated to contain a majority of the genes in certain species such as chickens $(14,15)$, and comparative genomic analysis has shown that genetic information in microchromosomes is conserved across multiple classes of chromosomes $(16,17)$. Thus, the genomic characterization of lizard microchromosomes may provide an important experimental model for comparative analysis of their unique traits, including tail regeneration. In this context, Alfoldi et al. not only provided lizard whole genome sequences, but also revealed that $A$. carolinensis microchromosomes are highly syntenic with chicken microchromosomes, although they exhibit some regional variation in GC content and a lower repeat content than do avian microchromosomes. Alfoldi et al. suggested that the synteny of microchromosomes in $A$. carolinensis and chickens may imply that these animals arose from a common reptilian ancestor, while other microchromosomes in the chicken are unique to the chicken lineage. The nucleotide composition of the $A$. carolinensis genome is as homogenous as is the frog genome, and this homogeneity is a distinctive property of the lizard compared to avian amniotes. In fact, Fujita et al. showed that $A$. carolinensis has the most compositionally homogeneous genome of all amniotes, with a degree of homogeneity exceeding that of Xenopus (18). They also found that the $A$. carolinensis genome contains a reduced size and number of isochores compared to that of humans or chickens. Since vertebrate genomes are mosaics of isochores and major changes among the amniotes are associated with the appearance of GC-rich isochores in avian and mammalian genomes (19), further study of the structural and compositional differences between lizards and other amniotes may give a hint of the mechanisms of tissue regeneration. In addition to compositional homogeneity and a reduced number of isochores, the $A$. carolinensis genome contains a high number of transposons. Transposons are the segments of DNA that can move themselves to new locations within the chromosomes of individual cells and create mutations by insertions, deletions, and translocations of genes. By doing this, transposons can create or reverse mutations in genomes, and accumulated transposition events produce interspersed repeats within genomes, ultimately facilitating the development of new genes by blocking gene conversion (20). Therefore, the high number of transposons in lizards may give them a flexible genome that can efficiently respond to unexpected environmental changes.

\section{COMPARATIVE ANALYSES OF THE GENETIC ELEMENTS RESPONSIBLE FOR TAIL REGENERATION OF LIZARDS}

The availability of the $A$. carolinensis genome sequence will play an important role in understanding the evolution of mammalian genomes and possible explanations for important branches of the evolutionary tree of vertebrates. However, the genome sequence itself may not give direct evidence for unique traits such as adhesive setae, nocturnal vision, and natural limb regeneration, and bioinformatic comparative analysis involving genomics, transcriptomics, and proteomics should be performed to investigate those mechanisms in lizards.

Very recently, Liu et al. published the full genome of Gekko japonicus, another species of lizard, and investigated the genetic elements related to its unique physiological processes including tissue regeneration (21). They obtained a genome sequence of $2.55 \mathrm{~Gb}$ and identified a total of 22,487 genes from G. japonicus. Of those genes, they selected 155 genes that are possible candidates for regeneration by comparing homologues in different species which are known to be involved in the biological processes necessary for tissue regeneration such as wound healing, cellular proliferation, and migration. Although functional analysis of each gene should be performed, positive selection for tail regeneration in $G$. japonicus seems to be feasible since the selected genes include some that were previously shown to be involved in the regeneration of various tissues in different species, such as prostacyclin synthase (PTGIS) and prostaglandin-endoperoxide synthase (PTGS1) $(22,23)$. These are enzymes involved in prostaglandin biosynthesis, and a recent report showed that inhibition of prostaglandin-degrading enzyme accelerates repair of various tissues such as bone marrow, colon, and 
liver, suggesting a key role of prostaglandin in tissue regeneration (24). Thus, that data will be of great value in understanding tissue regeneration, although further genomic sampling of other reptiles and amniotes should be done. However, it may be insufficient to only categorize the gene pool based on the genomic sequence itself. In order to discover key regeneration factors, it may be necessary to identify the genes that are differentially expressed during the regeneration period.

For such purposes, the first transcriptomic analysis of regenerating tails has recently been attempted by performing RNA-Seq on $A$. carolinensis (25). The researchers identified 326 genes with altered RNA levels associated with tail regeneration. Bioinformatic analysis showed that the genes are categorized into regeneration-related processes such as myogenesis, cellular adhesion, and the immune response. For example, the selected genes include the members of the Wnt and MAPK/FGF pathways, which are key molecules involved in tissue regeneration in a variety of organisms (26-30), giving clues to the veracity of that data. In addition to well-known regeneration factors, the researchers also obtained 22 novel genes that are differentially expressed during regeneration. Together with the well-known genes, characterization of these novel genes may provide valuable data sets for studying regenerative processes and related signaling pathways.

In addition to the above genomic and transcriptomic approaches, proteomic analysis has also been done to study the mechanisms of tail regeneration. The first gene identified as a dedifferentiation factor from proteomic studies in lizards was a lactoferrin, reported by Bae et al. in 2014 (31). Many case of tissue regeneration in lower animals involve cellular dedifferentiation to form a blastema, followed by redifferentiation and subsequent regenerative proliferation (32).
In fact, the first step in tissue regeneration is formation of a mound with specialized wound epithelium over the site of amputation $(32,33)$. This tissue undergoes dedifferentiation to form a blastema at the site of injury and subsequently re-enter the cell cycle, thus reconstructing complex structures through cellular proliferation and specialized differentiation.

According to the previous report, tail regeneration of the lizard (Eublepharis macularius) is divided into a wound healing phase, a dedifferentiation phase, and a redevelopment stage (32). To identify dedifferentiation factors, Bae et al. isolated proteins from the collected tail-tip tissues at each stage and analyzed the differential expression of the proteins by 2D-electrophoresis. Among a total of 292 proteins which are differentially expressed during tail regeneration, they initially selected 18 proteins which underwent increased expression during the dedifferentiation period and decreased expression in the redifferentiation phase. Protein homology analysis showed that these proteins are functionally categorized into cellular metabolism, protease inhibitor, cytoskeletal, and cellular differentiation factors. Of those 18, they identified lactoferrin as a dedifferentiation factor based on its high frequency and expression pattern. Functional studies with lactoferrin showed that it was specifically expressed in embryonic stem cells and induced pluripotent stem cells (iPSCs) in both mice and humans, and the addition of lactoferrin promoted the efficiency of iPSC generation. Furthermore, it was found that lactoferrin induces the expression of Klf4, a well-known factor involved in iPS generation (Fig. 1). Thus, it seems that lactoferrin promotes dedifferentiation by induction of endogenous Klf 4 expression. Lactoferrin is a multifunctional protein involved in various processes associated with wound healing, including cellular proliferation, migration, and survival (34-36). Interestingly,
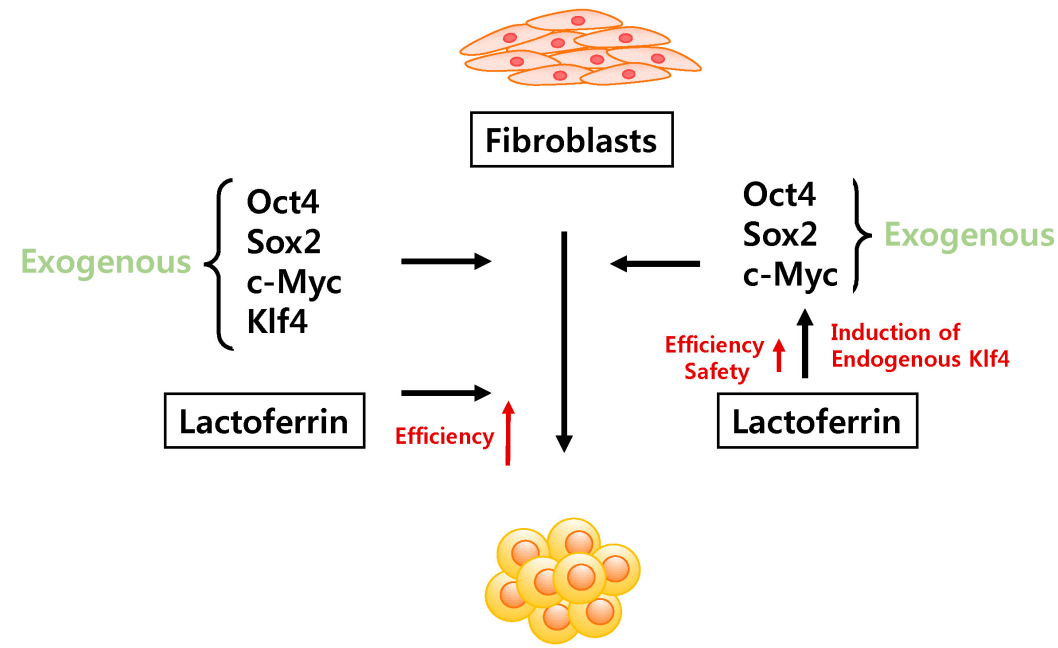

iPS
Fig. 1. Functional effects of LF on iPSC generation. Addition of LF to the culture medium during in vitro dedifferentiation increases the efficiency of iPSC generation compared with a conventional method in which only four genes (Oct4, Sox2, Klf4, and c-Myc) are introduced. Latoferrin can increase the efficiency of iPSC generation even without forced expression of KIf4 by induction of endogenous Klf4. 
lactoferrin is the sole natural tryptase inhibitor in humans. Tryptase is a trypsin-like serine protease secreted exclusively from the secretory granules of activated mast cells (37). Mast cells have been implicated in regenerative wound healing processes (38), and their proteases and inhibitors have been shown to contribute to the coordinated healing of cutaneous wounds (39). In fact, lactoferrin has been reported to be involved in regeneration of various tissues such as bone, cornea, and skin (40-43). Thus, it will be interesting to study the role of lactoferrin as a tryptase inhibitor in dedifferentiation in mammals.

\section{DISCUSSION AND PERSPECTIVES}

It is likely that all animals have the capacity to regenerate damaged body parts, although the degree of regeneration seems to be different in different species. Regeneration is more vigourous in invertebrates than it is in vertebrates. Indeed, many invertebrates, such as hydra, planarians, and starfish, have bidirectional regeneration capcity, so they can generate two sets of the same animal by regrowing missing parts, while regeneration processes in vertebrates occur unidirectionally, in which the animal reproduces only damaged parts at the site of injury (Fig. 2). Amongst vertebrates, fishes and amphibians have the greatest regenerative capacities, and amniotes such as reptiles, birds, and humans, seem to have lost the capability to regenerate, although many lizards can reproduce their tails. In lower vertebrates, natural regeneration occurs mainly by virtue of the intrinsic plasticity of mature tissues, which involves cellular proliferation, migration of remaining parts, and regrowth of damaged or missing parts. The most prominent event in tissue regeneration in lower vertebrate may be formation of a blastema. The blastema shares many characteristics with stem cells, and can eventually redevelop into various tissues, including muscle, skin, bone, and blood vessels, that were originally present at the damaged site. The blastema is formed through the dedifferentiation process, and this step is omitted in the higher vertebrates such as birds and mammals. Thus, it could be that the lack of regenerative capacity in birds and mammals may be evolutionarily related to loss of the capacity to dedifferentiate. In fact, mammals share many key factors for regeneration with lower animals, such as fibroblast growth factor (FGF), Wnt/beta-catenin, and bone morphogenic protein (BMP)/Msx signaling, which are known to be involved in wound healing and cellular proliferation (26-30, 44). Through such processes, mammals can repair damaged tissues to some extent. Nevertheless, mammals have little regenerative capacity compared to lower animals, probably because they lack the capability to dedifferentiate. Damage to human organs, such as the heart, brain, and liver, often leads to serious pathological conditions. Although stem cell-based transplantation could be clinically performed, additional strategies may be required for proper treatment of organ injuries in humans. Thus, study of the mechanisms of blastema formation and the development of protocols for mammalian dedifferentiation will be a breakthrough for regenerative medicine and stem cell biology. As a matter of fact, mammalian cells have been known to undergo dedifferentiation in vitro by enforced expression of Oct4, Sox2, Klf4, and c-Myc $(45,46)$. Although this iPSC strategy is an innovative tool in human tissue regeneration and stem cell therapeutics, it has drawbacks including low efficiency and uncertain safety (47). For example, use of oncogenes such as Klf4 and c-Myc in iPSC generation raised concerns about the safety of iPSCs for practical applications. Although other substitutes such as Nanog and Lin28 have been suggested (48), these oncogenes may be regarded as indispensable to the efficiency of dedifferentiation $(49,50)$. However, recent discoveries in proteomics showed that lactoferrin can substitute for Klf4, and even provide greater efficiency for dedifferentiation of human fibroblasts. Although lactoferrin by itself is not enough to replace all the oncogenes necessary for dedifferentiation of human cells, and further identification of other factors should be performed, this finding indicates that comparative studies of lizards would be a promising strategy to reveal the mechanisms of regeneration.

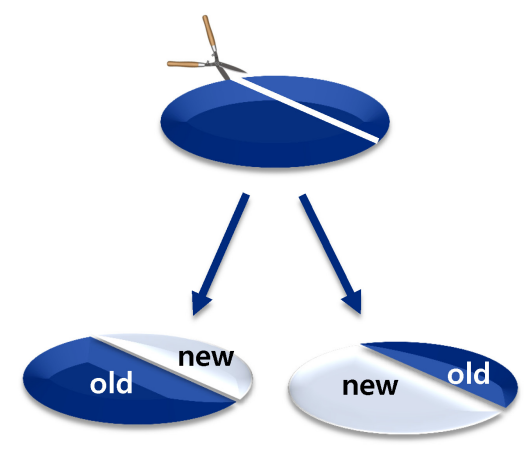

[Bidirectional regeneration]

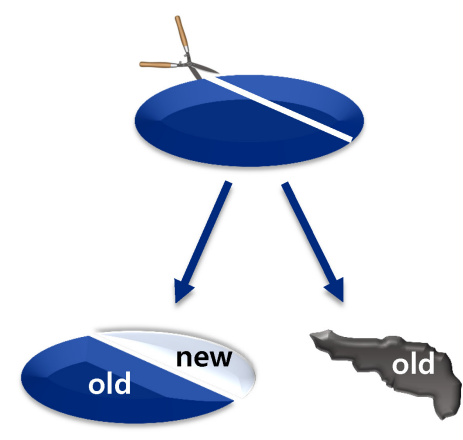

[Unidirectional regeneration]
Fig. 2. Natural regeneration in lower animals. Regenerative invertebrates, such as hydra, planarians, and starfishes, have bidirectional regeneration capcity, so that they can generate two sets of the same animal from both side of a damaged site. Regeneration processes in vertebrates, such as frogs, zebrafish, and salamanders, occurs unidirectionally, meaning that the animal reproduces only damaged parts at the site of an injury. 

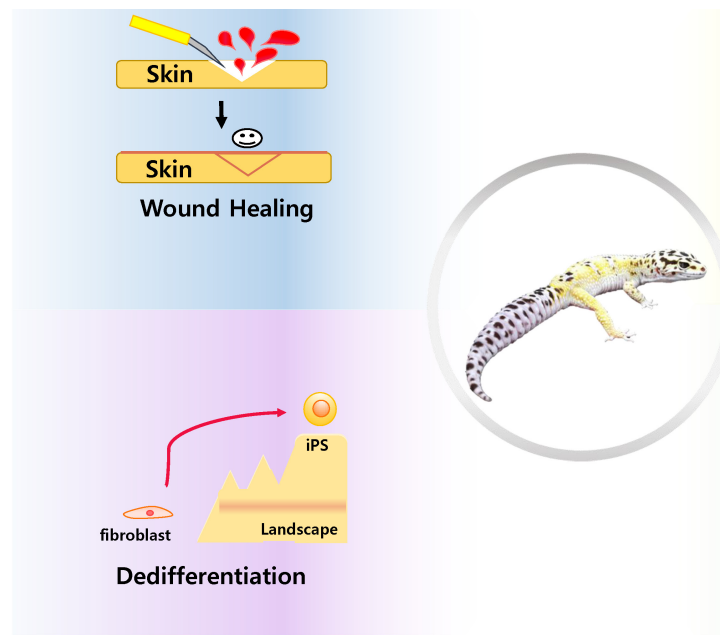

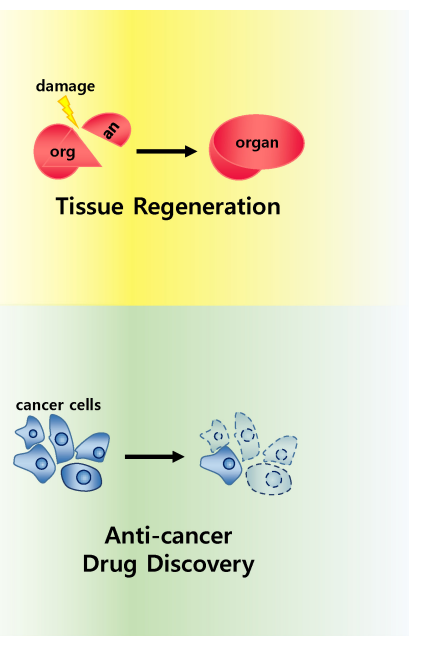

Fig. 3. Applications of lizard biology. Comparative analysis of the lizard genome may provide new insights into the evolutionary strategies of lower animals that are responsible for tissue regeneration, and may be a valuable source for the development of various fields including iPSC technology, medicinal biology, and industrial biology.
Now, we have a big picture of the lizard genome in hand. Elucidation of the similarities and differences between genomes of regenerative and non-regenerative animals will facilitate an understanding of regenerative biology, bring a new perspective to the development of regenerative medicine, and suggest clinical strategies for the treatment of injured human organs. In addition, the data from these approaches may contribute to various fields including the cosmetics industry and anti-cancer drug discovery (Fig. 3).

\section{ACKNOWLEDGEMENTS}

This work was supported by Dong-A University.

\section{REFERENCES}

1. Franco C, Soares R, Pires E et al (2013) Understanding regeneration through proteomics. Proteomics 13, 686-709

2. Rink JC (2013) Stem cell systems and regeneration in planaria. Dev Genes Evol 223, 67-84

3. Baranowitz SA, Maderson PF, Connelly TG (1979) Lizard and newt tail regeneration: a quantitative study. J Exp Zool 210, 17-37

4. Simkin J, Han M, Yu L, Yan M, Muneoka K (2013) The mouse digit tip: from wound healing to regeneration. Methods Mol Biol 1037, 419-435

5. Laurin M, Reisz RR (1995) A reevaluation of early amniote phylogeny. Zool J Linn Soc 113, 165-223

6. Foucart T, Lourdais O, DeNardo DF Heulin B (2014) Influence of reproductive mode on metabolic costs of reproduction: insight from the bimodal lizard Zootoca vivipara. J Exp Biol 217, 4049-4056

7. Kabelik D, Magruder DS (2014) Involvement of different mesotocin (oxytocin homologue) populations in sexual and aggressive behaviours of the brown anole. Biol Lett 10, pii: 20140566

8. Jeong AJ, Chung CN, Kim HJ et al (2012) Gecko Proteins
Exert Anti-Tumor Effect against Cervical Cancer Cells Via PI3-Kinase/Akt Pathway. Korean J Physiol Pharmacol 16, 361-365

9. Kim GY, Park SY, Jo A et al (2015) Gecko proteins induce the apoptosis of bladder cancer 5637 cells by inhibiting Akt and activating the intrinsic caspase cascade. BMB Rep 48, 531-536

10. Alföldi J, Di Palma F, Grabherr M et al (2011) The genome of the green anole lizard and a comparative analysis with birds and mammals. Nature 477, 587-591

11. Axelsson E, Webster MT, Smith NG, Burt DW, Ellegren $\mathrm{H}$ (2005) Comparison of the chicken and turkey genomes reveals a higher rate of nucleotide divergence on microchromosomes than macrochromosomes. Genome Res 15, 120-125

12. Groenen MA, Cheng $H H$, Bumstead $N$ et al (2000) A consensus linkage map of the chicken genome. Genome Res 10, 137-147

13. Fillon $\vee$ (1998) The chicken as a model to study microchromosomes in birds: a review. Genet Sel Evol 30, 209-219

14. Maan S, Maan NS, Samuel AR, Rao S, Attoui H, Mertens PP (2007) Analysis and phylogenetic comparisons of full-length VP2 genes of the 24 bluetongue virus serotypes. J Gen Virol 88, 621-630

15. Burt DW (2002) Origin and evolution of avian microchromosomes. Cytogenet Genome Res 96, 97-112

16. Gordon L, Yang S, Tran-Gyamfi M et al (2007) Comparative analysis of chicken chromosome 28 provides new clues to the evolutionary fragility of gene-rich vertebrate regions. Genome Res 17, 1603-1613

17. Shetty S, Griffin DK, Graves JA (1999) Comparative painting reveals strong chromosome homology over 80 million years of bird evolution. Chromosome Res 7, 289-295

18. Fujita MK, Edwards SV, Ponting CP (2011) The Anolis lizard genome: an amniote genome without isochores. Genome Biol Evol 3, 974-984

19. Bernardi G (1993) The vertebrate genome: isochores and 
evolution. Mol Biol Evol 10, 186-204

20. Schimenti JC, Duncan CH (1984) Ruminant globin gene structures suggest an evolutionary role for Alu-type repeats. Nucleic Acids Res 12, 1641-1655

21. Liu Y, Zhou Q, Wang Y et al (2015) Cekko japonicus genome reveals evolution of adhesive toe pads and tail regeneration. Nat Commun 6, 10033

22. Rudnick DA, Perlmutter, DH, Muglia LJ (2001) Prostaglandins are required for CREB activation and cellular proliferation during liver regeneration. Proc Natl Acad Sci U S A 98, 8885-8890

23. Hsueh YC, Wu JM, Yu CK, Wu KK, Hsieh PC (2014) Prostaglandin $\mathrm{E}(2)$ promotes post-infarction cardiomyocyte replenishment by endogenous stem cells. EMBO Mol Med 6, 496-503

24. Zhang Y, Desai A, Yang SY et al (2015) Tissue regeneration: Inhibition of the prostaglandin-degrading enzyme 15-PGDH potentiates tissue regeneration. Science 348, aaa2340

25. Hutchins ED, Markov GJ, Eckalbar WL et al (2014) Transcriptomic analysis of tail regeneration in the lizard Anolis carolinensis reveals activation of conserved vertebrate developmental and repair mechanisms. PLoS One 9, e105004

26. Geng R, Noda T, Mulvaney JF, Lin VY, Edge AS, Dabdoub A (2016) Comprehensive Expression of Wnt Signaling Pathway Genes during Development and Maturation of the Mouse Cochlea. PLoS One 11, e0148339

27. Hamilton PW, Sun Y, Henry JJ (2016) Lens regeneration from the cornea requires suppression of $\mathrm{Wnt} / \beta$-catenin signaling. Exp Eye Res 145, 206-215

28. Houschyar KS, Momeni A, Pyles MN Maan ZN, Whittam AJ, Siemers $F$ (2015) Wnt signaling induces epithelial differentiation during cutaneous wound healing. Organogenesis 11, 95-104

29. Pastuhov SI, Hisamoto N, Matsumoto K (2015) MAP kinase cascades regulating axon regeneration in $\mathrm{C}$. elegans. Proc Jpn Acad Ser B Phys Biol Sci 91, 63-75

30. Ornitz DM, Marie PJ (2015) Fibroblast growth factor signaling in skeletal development and disease. Genes Dev 29, 1463-1486

31. Bae KS, Kim SY, Park SY et al (2014) Identification of lactoferrin as a human dedifferentiation factor through the studies of reptile tissue regeneration mechanisms. J Microbiol Biotechnol 24, 869-878

32. McLean KE, Vickaryous MK (2011) A novel amniote model of epimorphic regeneration: the leopard gecko, Eublepharis macularius. BMC Dev Biol 11, 50

33. Brockes JP and Kumar A (2008) Comparative Aspects of Animal Regeneration. Annu Rev Cell Dev Biol 24, 525-549

34. Takayama Y, Aoki R (2012) Roles of lactoferrin on skin wound healing. Biochem Cell Biol 90, 497-503

35. Tang L, Wu JJ, Ma Q et al (2010) Human lactoferrin stimulates skin keratinocyte function and wound re-epithelialization. Br J Dermatol 163, 38-47

36. Tang L, Cui T, Wu JJ Liu-Mares W, Huang N, Li J (2010) A rice-derived recombinant human lactoferrin stimulates fibroblast proliferation, migration, and sustains cell survival. Wound Repair Regen 18, 123-131

37. Gan X, Liu D, Huang P, Gao W, Chen X, Hei Z (2012) Mast cell-releasing tryptase triggers acute lung injury induced by small intestinal ischemia-reperfusion by activating PAR-2 in rats. Inflammation 35, 1144-1153

38. Gourevitch D, Kossenkov AV, Zhang Y et al (2014) Inflammation and its correlates in regenerative wound healing: an alternate perspective. Adv Wound Care (New Rochelle) 3, 592-603

39. McCarty SM, Percival SL (2013) Proteases and Delayed Wound Healing. Adv Wound Care (New Rochelle). 2, 438-447

40. Li W, Zhu S, Hu J (2015) Bone Regeneration Is Promoted by Orally Administered Bovine Lactoferrin in a Rabbit Tibial Distraction Osteogenesis Model. Clin Orthop Relat Res 473, 2383-2393

41. Yoshimaki T, Sato S, Tsunori K et al (2013) Bone regeneration with systemic administration of lactoferrin in non-critical-sized rat calvarial bone defects. J Oral Sci 55, 343-348

42. Pattamatta U, Willcox M, Stapleton F, Garrett Q (2013) Bovine lactoferrin promotes corneal wound healing and suppresses IL-1 expression in alkali wounded mouse cornea. Curr Eye Res 38, 1110-1117

43. Takayama Y, Aoki R (2012) Roles of lactoferrin on skin wound healing. Biochem Cell Biol 90, 497-503

44. Makanae A, Mitogawa K, Satoh A (2016) Cooperative inputs of Bmp and Fgf signaling induce tail regeneration in urodele amphibians. Dev Biol 410, 45-55

45. Takahashi K, Yamanaka S (2006) Induction of pluripotent stem cells from mouse embryonic and adult fibroblast cultures by defined factors. Cell 126, 663-876

46. Takahashi K, Okita K, Nakagawa M, Yamanaka S (2007) Induction of pluripotent stem cells from fibroblast cultures. Nat Protoc 2, 3081-3089

47. Bayart E, Cohen-Haguenauer O (2013) Technological overview of iPS induction from human adult somatic cells. Curr Gene Ther 13, 73-92

48. Yu J, Vodyanik MA, Smuga-Otto K et al (2007) Induced pluripotent stem cell lines derived from human somatic cells. Science 318, 1917-1920

49. Nakagawa $M$, Kyanagi $M$, Tanabe $K$ et al (2008) Generation of induced pluripotent stem cells without Myc from mouse and human fibroblasts. Nat Biotechnol 26 101-106

50. Wernig M, Meissner A, Cassady JP, Jaenisch R (2008) c-Myc is dispensable for direct reprogramming of mouse fibroblasts. Cell Stem Cell 2, 10-12 